\title{
Composite Load Modeling via Measurement Approach
}

\author{
He Renmu, Ma Jin, Member, IEEE, and David J. Hill, Fellow, IEEE
}

\begin{abstract}
The accuracy of the load model has great effects on power system stability analysis and control. Based on our practice in China on modeling load from field measurements, this paper systematically develops a measurement-based composite load model. Principles guiding the load modeling practice are discussed based on detailed analysis on stochastic characteristics of the modeling procedure. The structure of the measurement-based composite load model is presented. A multicurve identification technique is described to derive parameters. The generalization capability of this built load model is also investigated in this paper. Two cases are studied to illustrate the accuracy of the developed load model on describing the load dynamic characteristics in the actual power system.
\end{abstract}

Index Terms-Load modeling, measurement approach, power system stability.

\section{NOMENCLATURE}

$\begin{array}{ll}R_{s} & \text { Stator winding resistance of motor (p.u.). } \\ X_{s} & \text { Stator leakage reactance of motor (p.u.). } \\ X_{m} & \text { Magnetizing reactance of motor (p.u.). } \\ R_{r} & \text { Rotor resistance of motor (p.u.). } \\ X_{r} & \text { Rotor leakage reactance of motor (p.u.). } \\ H & \text { Rotor inertia constant. } \\ A & \text { Torque coefficient in proportion to square of } \\ & \text { speed. } \\ B & \text { Torque coefficient in proportion to speed. } \\ C & \text { Constant torque coefficient. } \\ \omega & \text { Rotor speed. }\end{array}$

$I_{d}, I_{q} \quad d$-axis and $q$-axis stator current.

$U_{d}, U_{q} \quad d$-axis and $q$-axis bus voltage.

$E_{d}^{\prime}, E_{q}^{\prime} \quad d$-axis and $q$-axis transient EMF.

$E(x) \quad$ Expectation of the stochastic variable $x$.

$P_{Z}^{*}, P_{l}^{*}, P_{P}^{*} \quad$ Proportional coefficients of the constant impedance, constant current, and constant power in static active load.

Manuscript received April 18, 2005; revised August 16, 2005. This work was supported in part by the Chinese National Key Basic Research Special Fund under 2006.04CB217901, in part by the Hongkong Research Grant Council under CERG project CityU 1297/03E, in part by the NCEPU Fund (No.Dr200411), and in part by PCSIRT under IRT0515. This work was partly carried out when the second and third authors were at the Department of Electrical Engineering, City University of Hong Kong, Kowloon Tong, Hong Kong. Paper no. TPWRS-00224-2005.

H. Renmu and M. Jin are with the Key Laboratory of Power System Protection and Dynamic Security Monitoring and Control of Ministry of Education, North China Electric Power University, 102206 Beijing, China (e-mail: majin@tsinghua.org.cn).

D. J. Hill is with the Department of Information Engineering, the Australian National University, Canberra ACT 0200, Australia.

Digital Object Identifier 10.1109/TPWRS.2006.873130

$$
Q_{Z}^{*}, Q_{l}^{*}, Q_{Q}^{*}
$$

Proportional coefficients of the constant impedance, constant current, and constant reactive power in static reactive load.

\section{INTRODUCTION}

$\mathbf{T}$ HE importance of the load model to power system stability analysis and control has been well recognized [1]-[9]. Nevertheless, it is also widely known that the modeling load is quite difficult due to the uncertainty and the complexity of the load. Two approaches have been developed to build the load model, namely, the component-based method [10]-[12] and the measurement-based method [10], [13]-[18]. Both have advantages and disadvantages. No matter what approaches the utility takes to build the load model, there always exist three basic questions to be answered: First, what is the appropriate model structure of the aggregated load? Second, how should the load model parameters be identified? Third, does the load model thus built have good generalization capability? Obviously, load models based on sound answers to these three questions can reflect the aggregated load characteristic more accurately. Although all practices on load modeling are, more or less, directly or indirectly trying to answer these questions, to the authors' best knowledge, there are still no universal solutions to them, and more efforts still need to be dedicated in this field.

The structure of the load model has great impacts on the results of power system analysis [4]-[9]. It has been observed that different load models will lead to various, even completely contrary conclusions on system stability [19]-[21]. The traditional production-grade power system analysis tools often use the constant impedance, constant current, and constant power load model, namely, ZIP load model. However, simulation results by the modeling load as ZIP often deviate from the filed test results, which indicate the inefficiency of the ZIP load model. To capture the strong nonlinear characteristic of load under the recovery of the voltage, a load model with nonlinear structure was proposed by Hill [23]. Load structure in terms of nonlinear dynamic equations is later proposed by Karlsson and Hill [24]. Lin et al. [16] identify two dynamic load model structures based on measurements, stating that a second-order transfer function captures the load characteristics better than the first-order one. Lesieutre et al. [25] compare various dynamic load models and develop a PQ load model from a third-order induction motor model. The recent trend has tried to combine the dynamic load model with the static model [14], [17], [18], [20]-[22], [26]. Wang et al. [17] develop a load model as a combination of an RC circuit in parallel with an induction motor equivalent circuit. Shi and $\mathrm{He}$ [18] report their initial work on the measurement-based 
load modeling in China, where the composite load model structure is explored. By comparing the time-domain stability simulation results using various load models with corresponding data obtained from transient disturbance recorders, Kao et al. [20] conclude that the composite static and dynamic load model provides a more accurate representation. Similar conclusions are also validated in [21]. An interim composite load model with $80 \%$ static part and $20 \%$ part of induction motor model is proposed in [22] for WSCC system simulation. However, default parametric values are used in these models.

Except for the load model structure, the generalization capability of the load model is also very important. A load model built from one or two field tests may not be a good load model, since it may lack the ability to fit the new, fresh measured load curves. A good load model should approach the real load behavior in general under unseen voltage variations. Thus, estimating the generalization capability of the load model is a necessary part of the load modeling process; however, so far, this issue is not fully addressed during load modeling practice.

Based on our practices in China on load modeling from field measurements, this paper develops a measurement-based composite load model. This paper tries to answer the aforementioned three questions in a systematic way. The stochastic characteristic of measurement-based load modeling procedure will be analyzed in this paper, based on which principles guiding the load modeling process are proposed. These lay the theoretical foundation for the later work. Then, an improved composite load model structure is proposed and identified based on the data from the field measurements. Furthermore, the generalization capability of such load model is evaluated by applying the cross validation method.

This paper will be organized as follows. The general stochastic characteristic of measurement-based load modeling process will be discussed in Section II. In Section III, the composite load model structure to be identified from field measurements is presented, which answers the first question related to the model structure. The identification algorithm is presented in Section IV, answering the second question raised at the beginning of this paper. Section $\mathrm{V}$ focuses on estimating the generalization capability of the identified load model, which is certainly related to the answer to the third question. Two cases, based on our practice on building load model from field measurements in HuShiTai and JiXi substation, North East of China, will be discussed in Section VI. Section VI concludes this paper.

\section{Stochastic Characteristic of MeAsurement-BASED LOAD MODELING}

Since measurement-based load modeling is mathematically an identification problem, it can be sketched conceptually as shown in Fig. 1.

In Fig. $1, V$ is the bus voltage, the variation of which activates the respective variations of the load connected to the bus. The bigger the voltage variations are, the better the load characteristics can be stimulated. Generally, voltage dips larger than 0.1 p.u. are capable of stimulating load responses. Voltage dips

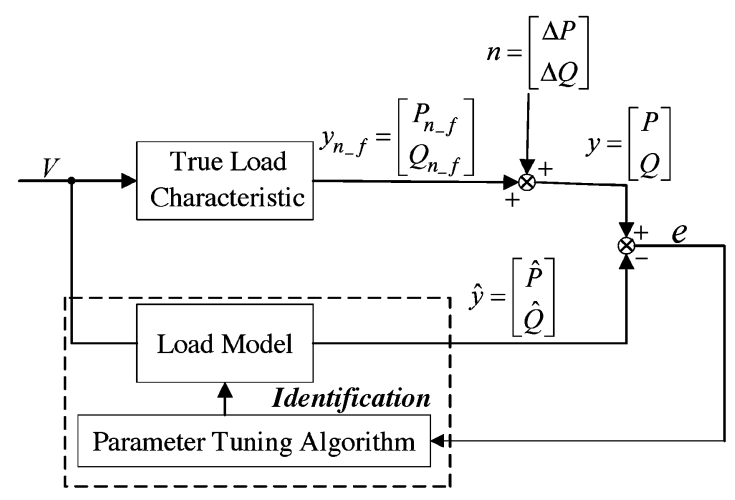

Fig. 1. Measurement-based load modeling.

larger than 0.3 p.u. have also been recorded in the field measurements but with less occurrence.

Under the voltage input, the output of the noise-free load characteristic should be $y_{n_{-} f}=\left[\begin{array}{l}P_{n_{-}} \\ Q_{n_{-}}\end{array}\right]$. To build the functional relationship of the load characteristic with respect to the voltage variation, it is generally assumed that during the very instant of the voltage disturbance, which may last only tens of ms, even shorter, the load variation is caused only by this voltage variation. However, there do exist possibilities that unmeasurable factors, such as some electric appliances are switched on, while some are switched off, coincidently happen in this short period. Thus, for each measurement, there always exists the small random variation of the load, which is not caused by the voltage disturbance. The random behavior from the power consumers at the moment of the voltage disturbance would lead to the uncertainty of the load, which is depicted in Fig. 1 as $n=\left[\begin{array}{l}\Delta P \\ \Delta Q\end{array}\right]$, added to the true load characteristics. Therefore, each measurement will produce an output $y=\left[\begin{array}{l}P \\ Q\end{array}\right]$, while the true load characteristic $y_{n_{-} f}$ is unobservable.

The identified load model under the bus voltage $V$ will produce the estimated load $\hat{y}=\left[\begin{array}{l}\hat{P} \\ \hat{Q}\end{array}\right]$. The difference between $y$ and $\hat{y}$ is fed back to the parameter tuning algorithm so that the parameters of the load model could be adjusted to minimize the expectation of the squared error, i.e.,

$$
\min E\left(e^{2}\right)
$$

which can be decomposed into two parts as follows:

$$
\begin{aligned}
E\left(e^{2}\right) & =E\left[(y-\hat{y})^{2}\right] \\
& =E\left[\left(y_{n_{-}} f-\hat{y}\right)^{2}\right]+E\left[n^{2}\right] .
\end{aligned}
$$

The first part of the right side of (2) is called the model error, which reflects the error between the true, but unmeasurable, load characteristic and load model output. The second part is the noise variance, which arises from the noise and is uncontrollable by optimizing the load model. Obviously, a good load model should keep the model error as small as possible. Under the ideal condition, if it is always zero, the load model thus built is completely a mirror of the true load. The model error can be further decomposed into the following two parts [27]:

$$
E\left[\left(y_{n_{-} f}-\hat{y}\right)^{2}\right]=\left[y_{n_{-} f}-E(\hat{y})\right]^{2}+E\left\{[\hat{y}-E(\hat{y})]^{2}\right\}
$$


which is often expressed as

$$
(\text { Model Error })^{2}=(\text { Bias Error })^{2}
$$$$
+(\text { Model Variance Error). }
$$

Bias error is closely related to the structure of the load model. For example, if the load were highly nonlinear, the linear model, no matter how high its order is, should never be expected to eliminate the bias error. Bias error describes the systematic deviation between the model and the real plant; thus, it is intrinsic to the model structure. Since any load model is only an aggregation of the numerous load components with various characteristics, the bias error always exist for the load model. We can only expect that the bias error of the load model is as small as possible. Generally, with the increased complexity of the model structure, and the increased number of parameters, the bias error monotonically decreases. From this point of view, we should choose a load model as complex as that permitted by the computation burden from both the identification and simulation procedure. However, it will be shown soon that too complex of a load model with lots of parameters will lead to a poor model generalization capability.

Except for the bias error, there exists the other term of model error, i.e., the model variance error. The model variance error arises because, in practice, the model parameters are estimated from a finite and noisy data set, and naturally, these parameters usually deviate from their optimal values. Since the bias error is intrinsic to the model structure, for newly measured data sets after the load model is built, the model error heavily depends on the model variance error. Thus, a bigger model variance error means a larger model error, which naturally leads to a lower predictability of the unseen data from the built model. It has been shown that in large data sets, the model variance error is linearly in proportion to parameter numbers of the model. The following expression approximately holds [27]:

$$
\text { Model Variance Error } \approx \sigma^{2} \frac{n_{p}}{N}
$$

where $\sigma^{2}$ is the variance of the noise. $n_{p}$ is the number of the parameters in the load model, and $N$ is the number of training data, i.e., the data samples used to estimate the model parameters. Equation (5) clearly shows that small variance of the noise and few number of parameters together with large number of training data will lead to the enhanced model generalization capability. This is in conformance with the Occam's razor principle, which states that from all models that can describe a process accurately, the simplest one is the best [27].

Based on the above analysis, the following three basic principles guiding our practice on load modeling are developed.

P1) The load model structure cannot be too simple. A too simple load model will lead to a big bias error, which is reflected as the big discrepancy between the model output and the recorded data from field measurements.

P2) The load model structure should not be too complex. A too complex load model will make a high model variance error. Therefore, while a complex load model may fit the training data well, nevertheless, it may have the poor generalization capability on voltage variations unseen in training data.

P3) The load model built on one or two field tests is unreliable, since it produces a high model variance error, inferring a low generalization capability. A good load model can only be built from the long-term observations on the load characteristics.

\section{Composite LoAd Model Structure}

Starting from the stochastic characteristic of the load modeling process, principles $\mathrm{P} 1$ and $\mathrm{P} 2$ in the last section provide good guidance on selecting the structure of the load model. However, in our practice on load modeling, except these two general principles, we find more specific aspects that must be attended. The first one is the intended applications of the load model. The load model we built is mainly for power system stability simulation and control. Thus, it should be easily integrated into the production-grade power system analysis tool. The second concern is the application range where the model is valid. A universal load model adapting to all situations does not exist. The reason lies in the fact that the load always consists of various components with different characteristics. Under extreme situations, such as those near the voltage collapse point or dramatic voltage dips sustaining for several seconds, some load components may show highly nonlinear characteristics, and some motors will drip off. A special load model should be built for these scenarios. The load model we want to develop is a general load model that should be valid under most scenarios in power system stability analysis. The last, but not the least, of the concerns is the customer acceptance. During our practice on load modeling, we find the black box load model is very difficult to be accepted by the system operators. System operators prefer a load model with clear physical interpretations.

Out of all these considerations, the motor in combination of ZIP has been chosen as the composite load model. As stated in the introduction part of this paper, there has been a trend to incorporate such a load model into the stability analysis. However, most of the work so far has centered on discussing the effects of such a model on system dynamics while using default parameters. The work to build this composite load model based on field measurements has not been systematically published yet and will be presented here. The equivalent circuit of this composite load model is shown in Fig. 2.

The model consists of a static part modeled as ZIP and a dynamic part, modeled as an equivalent circuit of the third-order motor dynamics. For this composite load model, a very important parameter to be identified is the level of the motor load in the total load, which is defined as

$$
K_{p m}=\frac{P_{\text {motor }}}{P_{0}}
$$

where $P_{0}$ is the initial active load on the bus, and $P_{\text {motor }}$ is the initial motor load. Another important parameter reflecting the effect of dynamic components on the total load is defined as

$$
M_{l f}=\frac{\left[\frac{P_{\text {motor }}}{S_{\text {MotorBase }}}\right]}{\left[\frac{V_{0}}{V_{\text {Base }}}\right]}
$$




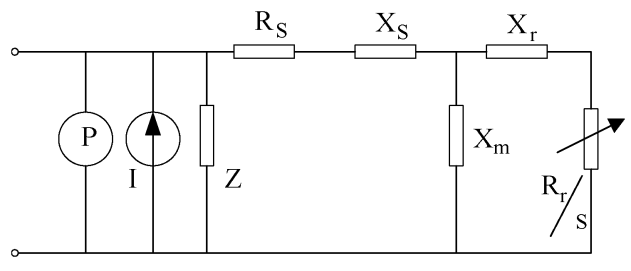

Fig. 2. Equivalent circuit of the composite load model.

where $S_{\text {MotorBase }}$ is the equivalent motor nominal capacity. $V_{0}$ and $V_{\text {Base }}$ are, respectively, the initial bus voltage and the voltage base. Obviously, the value of $S_{\text {MotorBase }}$ is closely related to the effects of the dynamic equivalent load on the total load. A big $S_{\text {MotorBase }}$ with a low $K_{p m}$ leads to a big equivalent motor but lightly loaded. On the other hand, a small $S_{\text {MotorBase }}$ with a high $K_{p m}$ leads to a small equivalent motor but heavily loaded. It can be expected that these two load models have quite different effects if applied to system stability analysis.

Following the definition of $K_{p m}$ and $M_{l f}$, the dynamic part of Fig. 2 can be described as

$$
\begin{aligned}
& \left\{\begin{array}{l}
\frac{d E_{d}^{\prime}}{d t}=-\frac{1}{T^{\prime}}\left[E_{d}^{\prime}+\left(X-X^{\prime}\right) I_{q}\right]-(\omega-1) E_{q}^{\prime} \\
\frac{d E_{q}^{\prime}}{d t}=-\frac{1}{T^{\prime}}\left[E_{q}^{\prime}+\left(X-X^{\prime}\right) I_{d}\right]+(\omega-1) E_{d}^{\prime} \\
\frac{d \omega}{d t}=-\frac{1}{2 H}\left[\left(A \omega^{2}+B \omega+C\right) T_{0}-\left(E_{d}^{\prime} I_{d}+E_{q}^{\prime} I_{q}\right)\right]
\end{array}\right. \\
& \left\{\begin{array}{l}
I_{d}=\frac{1}{R_{s}^{2}+X^{\prime 2}}\left[R_{s}\left(U_{d}-E_{d}^{\prime}\right)+X^{\prime}\left(U_{q}-E_{q}^{\prime}\right)\right] \\
I_{q}=\frac{1}{R_{s}^{2}+X^{\prime 2}}\left[R_{s}\left(U_{q}-E_{q}^{\prime}\right)-X^{\prime}\left(U_{d}-E_{d}^{\prime}\right)\right]
\end{array}\right.
\end{aligned}
$$

where

$$
\begin{aligned}
T^{\prime} & =\frac{X_{r}+X_{m}}{R_{r}} \\
X & =X_{s}+X_{m} \\
X^{\prime} & =X_{s}+\frac{X_{m} X_{r}}{X_{m}+X_{r}} \\
A+B+C & =1 .
\end{aligned}
$$

The first two equations in (8) describe flux decay dynamics, while the third equation is the inertia dynamics. The static part of Fig. 2 can be described as

$$
\left\{\begin{array}{l}
P_{s}^{*}=P_{Z}^{*}\left(\frac{V}{V_{0}}\right)^{2}+P_{I}^{*}\left(\frac{V}{V_{0}}\right)+P_{P}^{*} \\
Q_{s}^{*}=Q_{Z}^{*}\left(\frac{V}{V_{0}}\right)^{2}+Q_{I}^{*}\left(\frac{V}{V_{0}}\right)+Q_{Q}^{*} .
\end{array}\right.
$$

It satisfies

$$
\begin{aligned}
P_{Z}^{*}+P_{I}^{*}+P_{P}^{*} & =1-K_{p m} \\
Q_{Z}^{*}+Q_{I}^{*}+Q_{Q}^{*} & =1-\frac{Q_{\text {motor }}}{Q_{0}}
\end{aligned}
$$

where $Q_{0}$ is the initial reactive load on the bus, while $Q_{\text {motor }}$ is the initial reactive power consumed by the motor, which can be initialized based on the equivalent circuit in Fig. 2. The general form of this composite load model can be expressed as the following dynamics:

$$
\begin{aligned}
\dot{\mathbf{x}} & =f(\mathbf{x}, \mathbf{y}, \boldsymbol{\theta}) \\
\mathbf{0} & =g(\mathbf{x}, \mathbf{y}, \boldsymbol{\theta})
\end{aligned}
$$

where $\mathbf{x}$ and $\mathbf{y}$ refer, respectively, to the dynamic as well as the algebraic states of the equivalent load. $\boldsymbol{\theta}$ denotes the model parameters.

Table I presents the numerical interval of the load model parameters during identification. It has been observed that the re-
TABLE I

NUMERICAL INTERVEL OF THE LOAD MODEL PARAMETER

\begin{tabular}{|c|c|}
\hline Load Model & Parameters Range \\
\hline $\mathrm{Rs}$ & {$[0.1,0.35]$} \\
\hline $\mathrm{Xs}$ & {$[0.1,0.18]$} \\
\hline $\mathrm{Xm}$ & {$[2,3.8]$} \\
\hline $\mathrm{Rr}$ & {$[0.01,0.08]$} \\
\hline $\mathrm{Xr}$ & {$[0.5,1.5]$} \\
\hline $\mathrm{H}$ & {$[0.2,1]$} \\
\hline $\mathrm{A}$ & {$[0,1]$} \\
\hline $\mathrm{B}$ & {$[0.2,0.95]$} \\
\hline $\mathrm{K}_{\mathrm{pm}}$ & {$[0.2,0.65]$} \\
\hline $\mathrm{M}_{\mathrm{lf}}$ & {$[0.1,0.9]$} \\
\hline $\mathrm{P}_{\mathrm{p}}{ }^{*}$ & {$[0.1,0.9]$} \\
\hline $\mathrm{P}_{\mathrm{Z}}{ }^{*}$ & {$[0.1,0.9]$} \\
\hline $\mathrm{Q}_{\mathrm{Q}}{ }^{*}$ & {$[0.1,4]$} \\
\hline $\mathrm{Q}_{\mathrm{z}}{ }^{*}$ & \\
\hline
\end{tabular}

active portion of the load can be very voltage sensitive, which is attributed to the saturation of the distribution transformer by [7]. In addition to that, the voltage regulator in the distribution network may also lead to a high sensitivity of reactive load with respect to the voltage. Thus, in Table I, the permitted numerical interval for coefficients $Q_{Z}^{*}$ of the reactive portion is taken much larger than that for the coefficients $P_{Z}^{*}$ of the active portion, which means a more flexible model on reactive load. $P_{l}^{*}$ and $Q_{l}^{*}$ are also permitted to be negative in the equivalent load model to increase the model flexibility, since local power generation may exist.

After initial choices of the numerical intervals are made, they are further refined based on trials on many measured load data. If the numerical parameter intervals are inappropriate, the identification program will terminate when the preset maximum number of iterations is reached, leaving a big fitting error. Then the parameter numerical intervals are adjusted. With the wide application of the load characteristic recorder in various substations in China, the stable numerical intervals for parameter identification have been formed and applied so far, which guarantees the good performances of the identification process.

\section{Multicurve IDENTIFICATION}

According to the principle P3 in Section II, the load model identified from multiple data sets is more accurate than that built from one single test sample. Assume there have been $M$ recorded data available; then, for the $i$ th data, the root-meansquared error between the model output and the field measurement can be formulated as

$$
\begin{aligned}
e_{p i} & =\sqrt{\frac{\sum_{k=1}^{N} W_{P}(k)[\hat{P}(k)-P(k)]}{N}} \\
e_{q i} & =\sqrt{\frac{\sum_{k=1}^{N} W_{Q}(k)[\hat{Q}(k)-Q(k)]^{2}}{N}}
\end{aligned}
$$


where $N$ is the sampling points of this measurement. $\hat{P}(k)$, $\hat{Q}(k)$ are, respectively, the model output of active load and reactive load at the $k$ th step, while $P(k), Q(k)$ are the measured active load and reactive load at the $k$ th step. $W_{P}$ and $W_{Q}$ are weighting vectors for active and reactive load. Based on our practice, we choose

$$
W_{P}(k)=W_{Q}(k)=0.4 \frac{|U(k)-\bar{U}|}{\sum_{k=1}^{n}|U(k)-\bar{U}|}+\frac{0.6}{n}
$$

which clearly shows that sampling points with larger variance have bigger weight. Equations (13) and (14) are absolute fitting errors. Since the load value may vary in a great range among the measurements, the relative error is better to reflect the accuracy of the fitting, which can be defined as follows:

$$
\begin{aligned}
& \widetilde{e}_{p i}=\frac{e_{p i}}{\bar{P}_{i}} \\
& \widetilde{e}_{q i}=\frac{e_{q i}}{\bar{Q}_{i}}
\end{aligned}
$$

where

$$
\bar{P}_{i}=\frac{\sum_{k=1}^{N} P(k)}{N} \quad \bar{Q}_{i}=\frac{\sum_{k=1}^{N} Q(k)}{N}
$$

correspond to the average active load and reactive load for the $i$ th data.

Thus, the loss function for multicurve identification can be formulated as

$$
\min \frac{1}{M} \sum_{i=1}^{M} W_{i}\left(\widetilde{e}_{p i}+\widetilde{e}_{q i}\right)
$$

where $W_{i}$ are the weighting coefficients for different measurements. The choice of the weighting coefficients in (18) is based on the pre-analysis on the recorded data sample and varies from substation to substation. Obviously, (18) is an alternative form of (1) in Section II. Since (18) summarizes all the errors from each single measurement, minimizing objective (18) produces a load model fitting all the measurements at best, which is called by us an expectation composite load model.

The multicurve load modeling process is here formulated as a nonlinear optimization problem, which is to find the parameter set

$$
\left[R_{s}, X_{s}, X_{m}, R_{r}, X_{r}, H, A, B, K_{p m}, M_{l f}, P_{Z}^{*}, P_{P}^{*}, Q_{Z}^{*}, Q_{Q}^{*}\right]
$$

in the load model (6)to (11), minimizing the objective (18). There are two classes of optimization algorithms, in general, to solve this problem [27]. One approach is the nonlinear local optimization techniques, which include simplex search method, gradient-based method, and nonlinear least-square method; the other class is called nonlinear global optimization techniques, which include simulated annealing, genetic algorithms, and tabu search. The nonlinear local optimization technique has a fast convergence speed but relies heavily on the start guess on the parameter. Thus, it falls easily into a local optimum. On the other hand, since the nonlinear global optimization technique searches the optimum in the whole parameter space, it is unlikely to be stuck in some local optimum; nevertheless, the con- vergence speed is very slow. To enhance the efficiency of the identification process, a hybrid algorithm combining the genetic algorithm and simplex search method is implemented in our practice. The genetic algorithm is applied first to find regions in which the optimum may lie. Then the simplex search method is run to find the optimum.

\section{ESTIMATING THE GENERALIZATION CAPABILITY OF THE COMPOSITE LOAD MODEL}

As stated in the introduction part, after getting all the parameters of the composite load model, the load modeling process remains incomplete. For every model, it is important to study its capability to fit new, unseen data. Thus, we have to evaluate the generalization capability of the derived load model. In Section II, (5) gives a theoretical estimate when analyzing the model generalization capability. However, since the noise variance is actually unknown, (5) cannot be used in practice to evaluate the generalization capability of the load model.

Since the generalization capability of load model can only be found in new, unseen data, the whole data set can be divided into the training data set and the test data set. The load model identified on the training data set will be evaluated in the test data set. Based on its performance on the test data, a rough approximation on its generalization capability can be made. To fully utilize the data set and give a better approximation on the generalization capability of the derived load model, the cross-validation method is applied.

Assume there are $M$ data sets in total. The cross-validation procedure can be implemented in the following steps:

Step 1) Distribute these data equally into $L$ subsets in a random way, with $M / L$ data in each subset.

Step 2) Take one subset as the test data set, while all the data in the remaining $(L-1)$ subsets are treated as training data. Build the load model based on the training data.

Step 3) Validate the model built in Step 2) using the test data set, and the fitting error is denoted as $e_{i}^{v}$.

Step 4) If each set of $L$ sets has been chosen to be the test set, go to Step 5); otherwise, go back to Step 2).

Step 5) The error of the model for new, unseen data can be estimated as

$$
\hat{e}=\frac{1}{L} \sum_{i=1}^{L} e_{i}^{v}
$$

It has been noted in [27] that $\hat{e}$ can be used to obtain a good estimate of the model performance on fresh data. For final use, the model still should be trained on the whole available data set.

\section{CASe Study}

Our practice on measurement-based load modeling in the China power system can be dated back to 1985 . The first production-grade load characteristic recorder was applied in Cangzhou substation in 1995. So far, there have been 14 load characteristic recorders in operation around China. Among them, three are in South China, four are in North China, and 
TABLE II

LOAd MOdEl Parameter OF HuShitai Substation

\begin{tabular}{|c|c|c|}
\hline Load Model & Numerical Value & Variance \\
\hline $\mathrm{Rs}$ & 0.3441 & 0.00048145 \\
\hline $\mathrm{Xs}$ & 0.1781 & 0.00094501 \\
\hline $\mathrm{Xm}$ & 2.0424 & 0.0001687 \\
\hline $\mathrm{Rr}$ & 0.0322 & 0.00014986 \\
\hline $\mathrm{Xr}$ & 0.1765 & 0.00081106 \\
\hline $\mathrm{H}$ & 1.4569 & 0.00015055 \\
\hline $\mathrm{A}$ & 0.3475 & 0.00042412 \\
\hline $\mathrm{B}$ & 0.9216 & 0.0007566 \\
\hline $\mathrm{K}_{\mathrm{pm}}$ & 0.3382 & 0.00044861 \\
\hline $\mathrm{M}_{\mathrm{lf}}$ & 0.2688 & 0.0010084 \\
\hline $\mathrm{P}_{\mathrm{p}}{ }^{*}$ & 0.1031 & 0.00016689 \\
\hline $\mathrm{P}_{\mathrm{Z}}{ }^{*}$ & 0.6490 & 0.00011447 \\
\hline $\mathrm{Q}_{\mathrm{Q}}{ }^{*}$ & 1.8894 & 0.0004549 \\
\hline $\mathrm{Q}_{\mathrm{z}}{ }^{*}$ & 0.2757 & 0.0009818 \\
\hline Model Error & $3.9 \mathrm{e}-4$ & \\
\hline
\end{tabular}

seven are in Northeast China. More are expected to be applied soon in South and North China. The load model presented in this paper has been validated by various scenarios in the substation during its operation and proved to be accurate enough to simulate the true load behavior on power system stability analysis and control. This proposed load model has been taken by China Electric Power Research Institute (CEPRI) as a standard load model in their production-grade software package Power System Analysis Package (PSASP) and, thus, can be easily incorporated into system stability analysis.

In this section, two cases are presented to illustrate the accuracy of the developed model in describing the load dynamic characteristics.

\section{A. Measurement-Based Load Modeling in HuShiTai Substation}

HuShiTai power substation lies in Liao Ning province, China. The load characteristic recorder has been in operation since July 2003. Table II shows the load model parameters as well as their variances built on 90 measurements from the load recorder.

Observing Table II, we can find the identified load model has a bigger equivalent $R s$. That is because in the distribution network, the resistance of the distribution line normally has a bigger value. The model error shown in Table II is 3.9e-4, which demonstrates quite satisfactory fitting accuracy. Figs. 3-5 show the load dynamic characteristic in one disturbance on July 24 , 2003. The solid line is the field measurement, while the dashed line is the model output.

Figs. 3-5 clearly show that the load model describes the load characteristics under twice voltage variations well. It should be noted that due to the random and complex nature of the load, a load model can only be an aggregated one with approximation. Thus, a load model cannot fit the measured load curve point

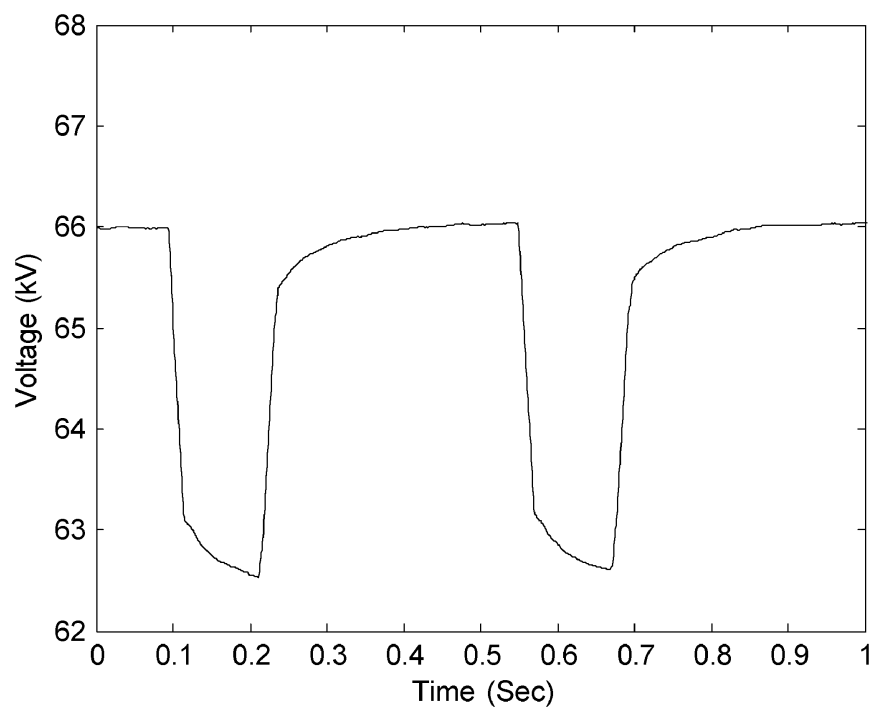

Fig. 3. Bus voltage of HuShiTai substation on 14:38:54, July 24, 2003.

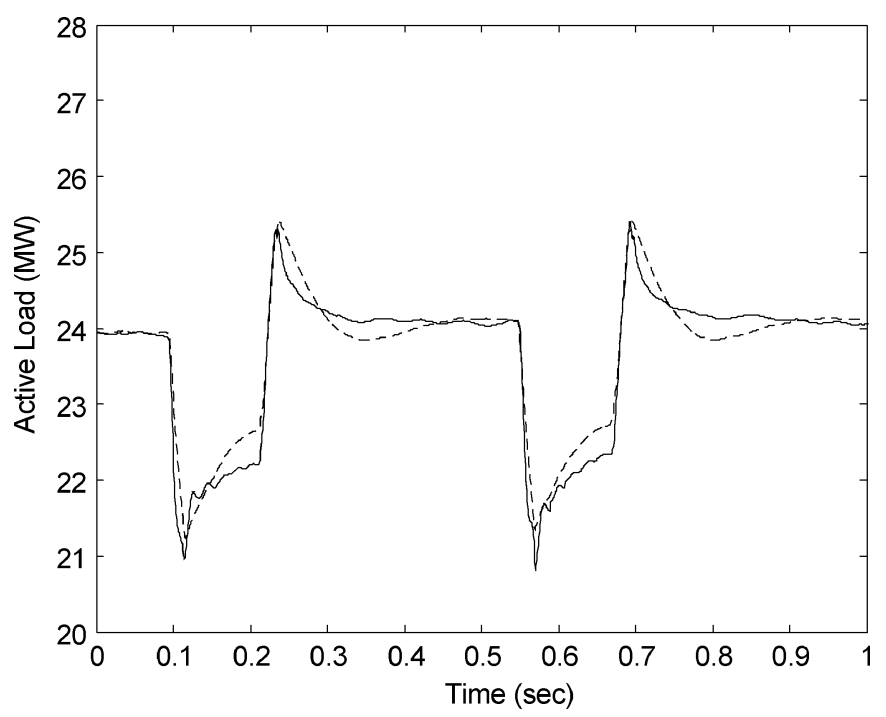

Fig. 4. Active load of both model output and the measurement.

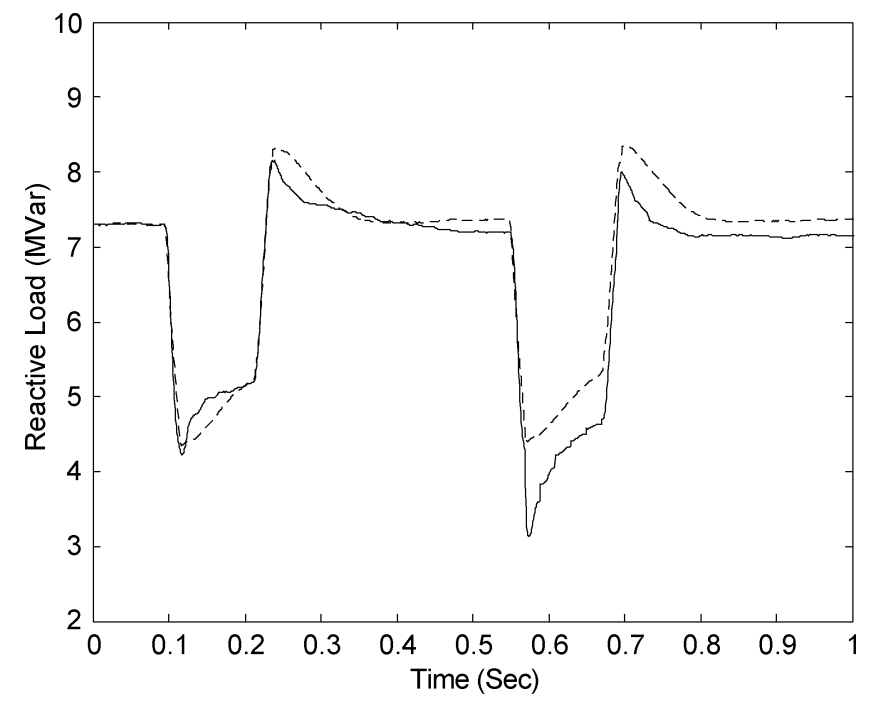

Fig. 5. Reactive load of both model output and the measurement.

by point; on the contrary, it should reflect the load characteristic in general. Actually, a load model, which fits one or two 
TABLE III

GENERALIZATION CAPABILITY ESTIMATE ON LOAD MODEL OF HUSHITAI SUBSTATION

\begin{tabular}{|l|l|l|}
\hline Data Subset & Training Error & Validation Error \\
\hline 1 & $4.163356 \mathrm{e}-004$ & $1.388901 \mathrm{e}-003$ \\
\hline 2 & $3.980500 \mathrm{e}-004$ & $1.316049 \mathrm{e}-003$ \\
\hline 3 & $4.501120 \mathrm{e}-004$ & $8.381140 \mathrm{e}-004$ \\
\hline $\begin{array}{l}\text { Estimate on the Model Error for } \\
\text { Unseen Data }\end{array}$ & $0.12 \%$ \\
\hline
\end{tabular}

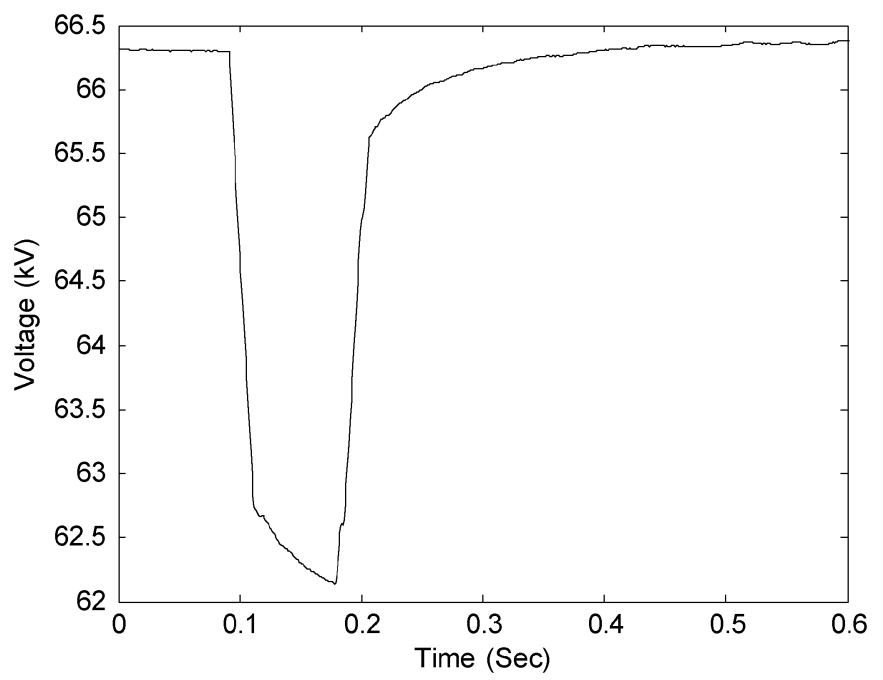

Fig. 6. Bus voltage of HuShiTai substation on 18:17:21, Aug. 21, 2003.

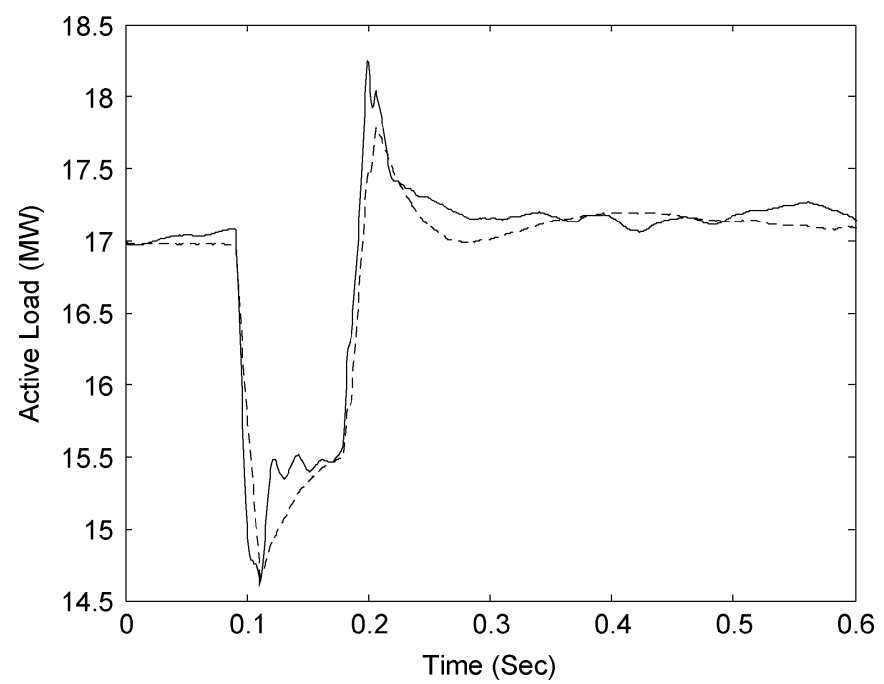

Fig. 7. Active load of both model predication and the measurement.

measured data too accurately, has normally poor generation capability when fitting other measured curves. These are called over-fitting problems. To give an estimate on the generalization capability of the built load model in Table II, we distribute all the data randomly into three subsets with 30 data in each set. Then the cross-validation method in Section V is applied. The results are shown in Table III.

Thus, the load model in Table I has reached an accuracy level for predicting the unseen data, which demonstrates a very good generalization capability. Figs. 6-8 show the model output

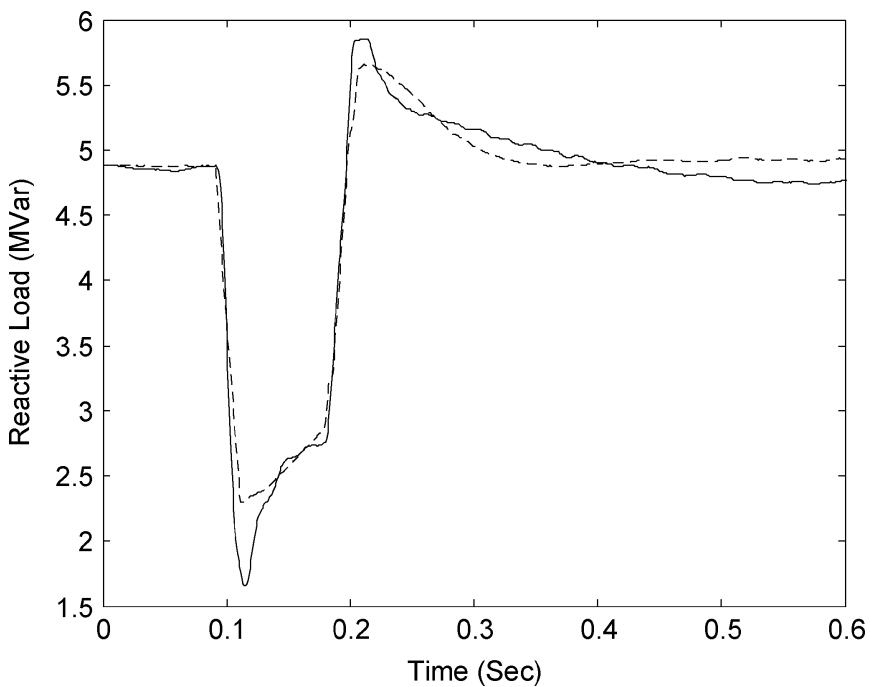

Fig. 8. Reactive load of both model predication and the measurement.

TABLE IV

LOAD MOdEl PARAMETER OF JiXI SUbSTATION

\begin{tabular}{|c|c|l|}
\hline Load Model & Numerical Value & Variance \\
\hline $\mathrm{Rs}$ & 0.2010 & 0.00080033 \\
\hline $\mathrm{Xs}$ & 0.1791 & 0.00013736 \\
\hline $\mathrm{Xm}$ & 2.0141 & 0.00041536 \\
\hline $\mathrm{Rr}$ & 0.0424 & 0.00012675 \\
\hline $\mathrm{Xr}$ & 0.1507 & $1.3653 \mathrm{e}-005$ \\
\hline $\mathrm{H}$ & 1.4529 & 0.00031992 \\
\hline $\mathrm{A}$ & 0.6267 & 0.00018455 \\
\hline $\mathrm{B}$ & 0.4471 & 0.00050259 \\
\hline $\mathrm{K}_{\mathrm{pm}}$ & 0.4676 & $2.5813 \mathrm{e}-005$ \\
\hline $\mathrm{M}_{\mathrm{lf}}$ & 0.3976 & $1.6333 \mathrm{e}-005$ \\
\hline $\mathrm{P}_{\mathrm{p}}{ }^{*}$ & 0.1094 & 0.00040187 \\
\hline $\mathrm{P}_{\mathrm{Z}}{ }^{*}$ & 0.7463 & 0.00019959 \\
\hline $\mathrm{Q}_{\mathrm{Q}}{ }^{*}$ & 3.1588 & 0.00079837 \\
\hline $\mathrm{Q}_{\mathrm{z}}{ }^{*}$ & 0.3071 & 0.00016075 \\
\hline $\mathrm{Model} \mathrm{Error}$ & $1.5 \mathrm{e}-3$ & \\
\hline & & \\
\hline & & \\
\hline & & \\
\hline
\end{tabular}

(dashed line) in a disturbance on Aug. 21, 2003. Also shown in Figs. 6-8 are field measurements on load dynamic characteristic (solid line). This disturbance is not included in the 90 data set for load model identification. Thus, it is one unseen datum when the load model of Table II is built. From Figs. 6-8, it can be seen that the model of Table II describes this unseen load characteristic quite accurately.

\section{B. Measurement-Based Load Modeling in JiXi Substation}

JiXi substation lies in Heilongjiang province. By application of the presented load modeling procedure, the load model can be built as shown in Table IV.

Figs. 9-11 show a disturbance recorded on March 10, 2003, which has been included in the training data set to develop the 


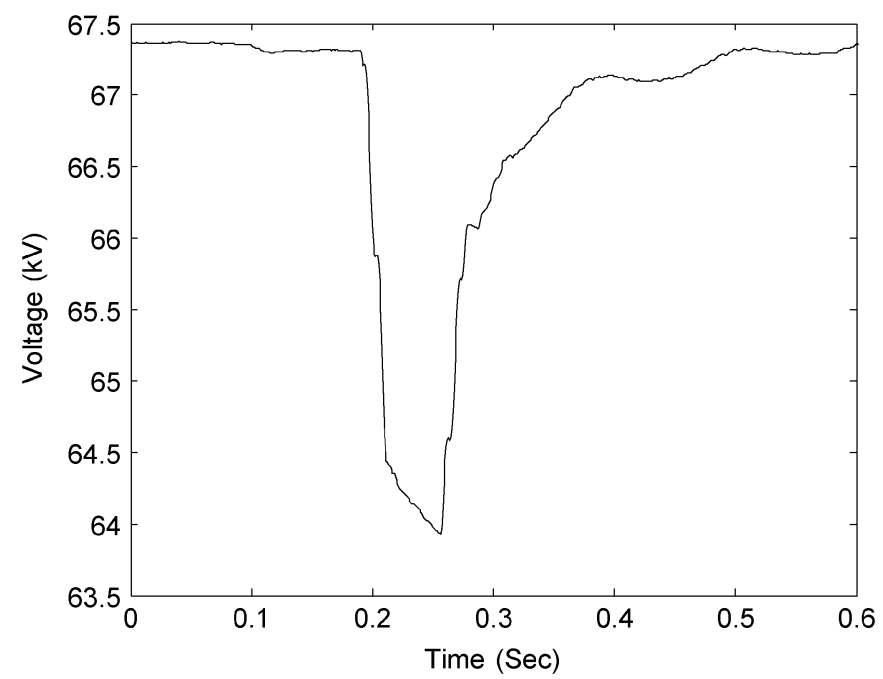

Fig. 9. Bus voltage of JiXi substation on 5:54:27, March 10, 2003.

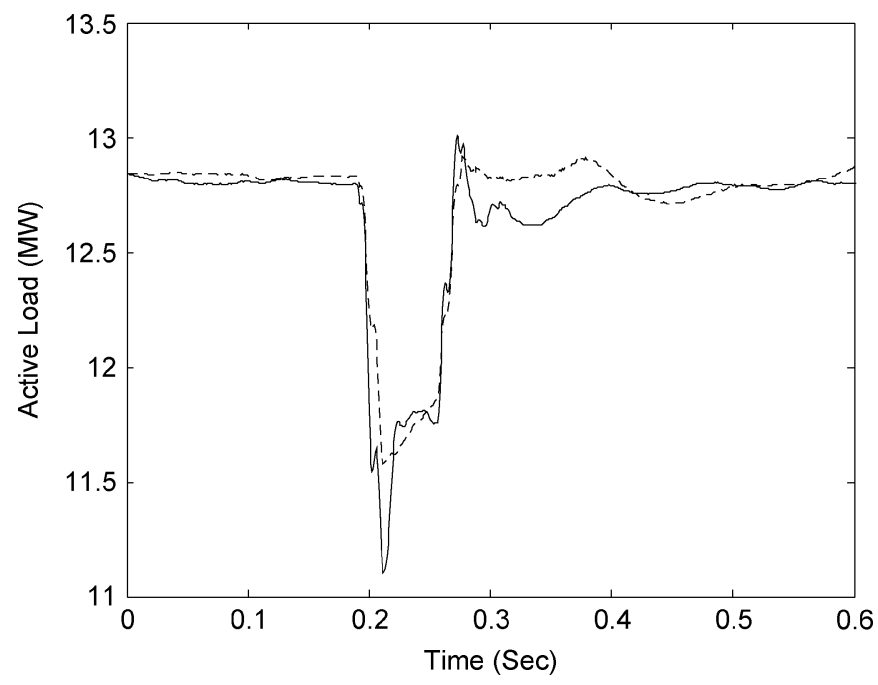

Fig. 10. Active load of both model output and the measurement.

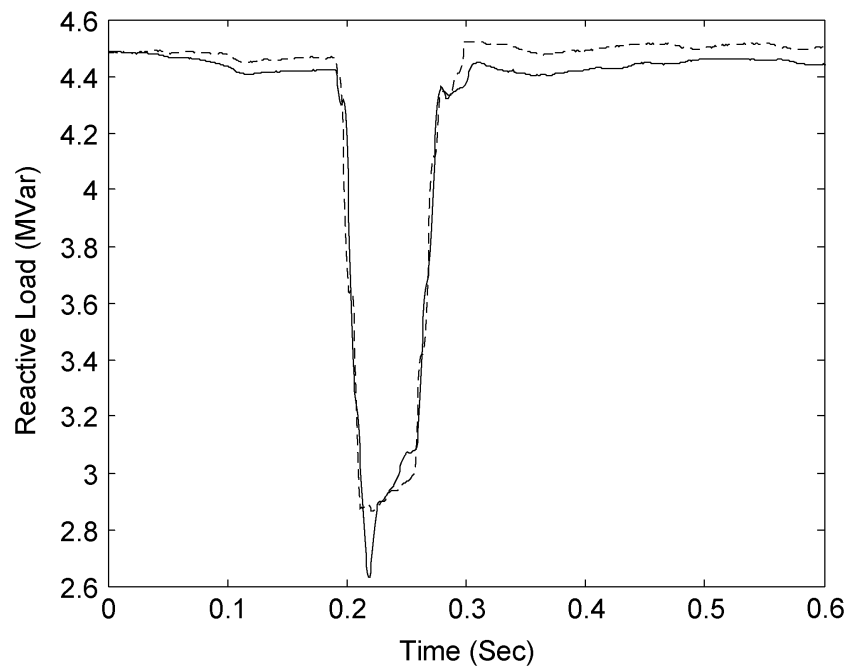

Fig. 11. Reactive load of both model output and measurement.

model of Table IV. It can be seen clearly that the model can describe the measured load dynamic well.

Figs. 12-14 show one disturbance recorded on March 1, 2003. This disturbance is not included in the training data set

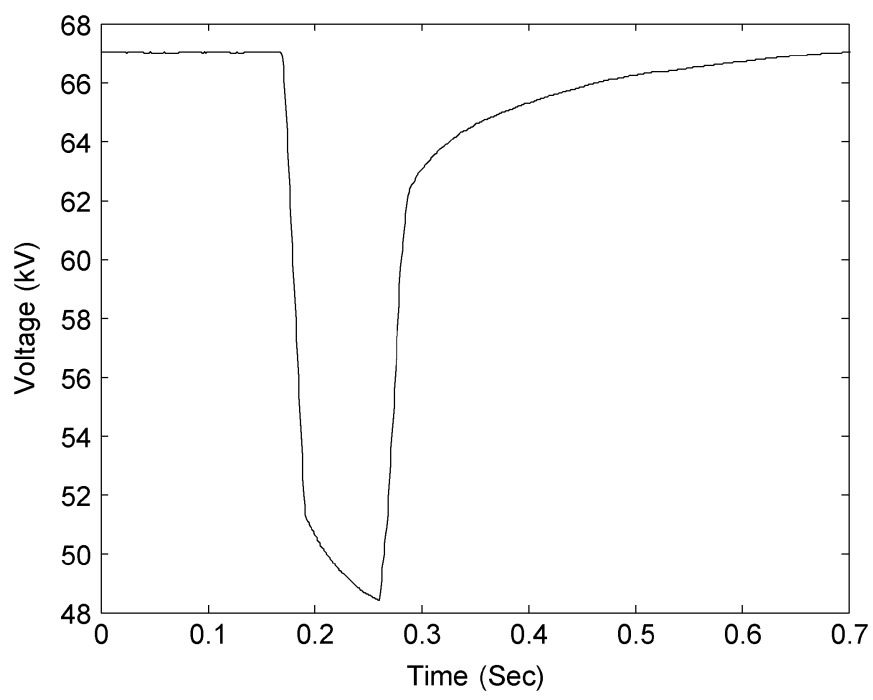

Fig. 12. Bus voltage of JiXi substation on 10:29:11, March 1, 2003.

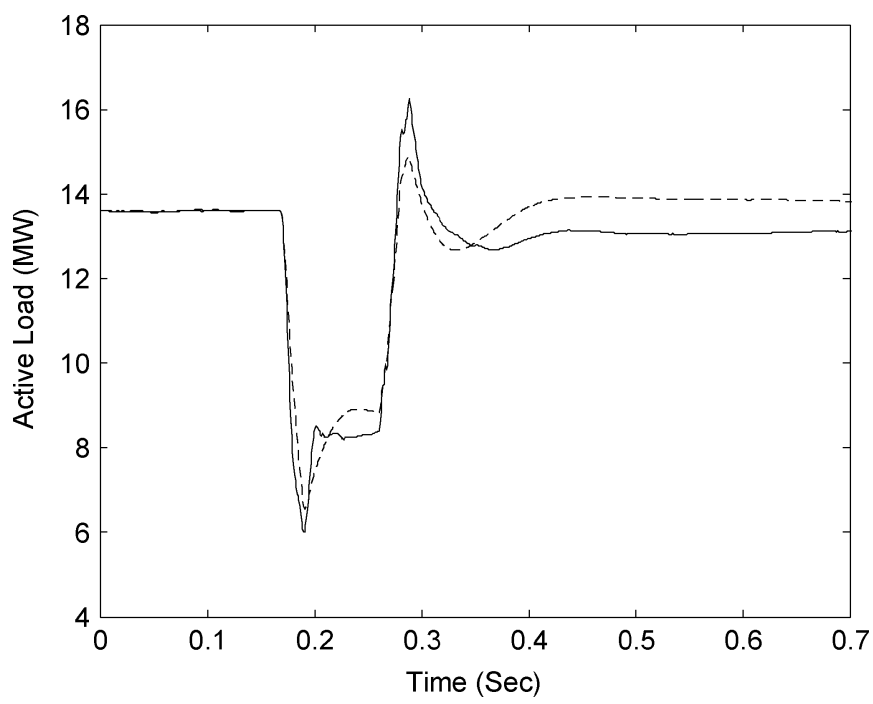

Fig. 13. Active load of both model predication and the measurement.

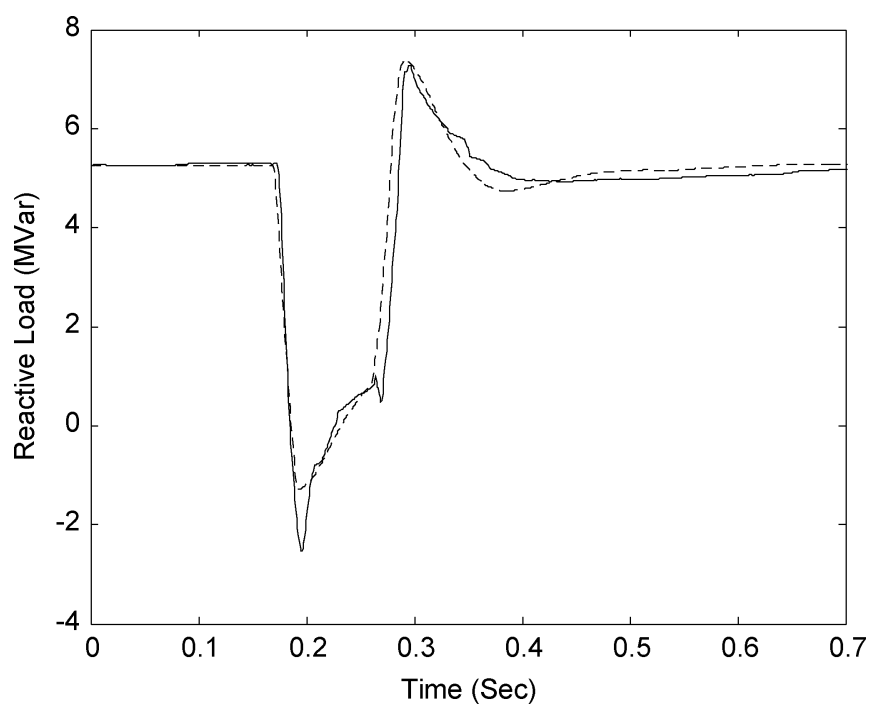

Fig. 14. Reactive load of both model predication and the measurement.

to build load model of Table IV due to the randomness when forming the training data set. Compared with Fig. 9, Fig. 12 shows a much lower and longer bus voltage dip during this 
disturbance. The voltage dip reaches nearly 0.3 p.u. Comparing the model output (dashed line) and the real load characteristics (solid line) in Figs. 13 and 14, we can see, although there exist deviations between the model output and the measured load characteristic, the load model of Table IV gives an acceptable performance. This shows a strong generalization capability of this load model.

\section{CONCLUSION AND FUTURE WORK}

This paper systematically develops a measurement-based composite load model. The composite load model has the structure of a motor in combination with a static ZIP type, and all its parameters are identified from field measurements based on the multicurve identification technique presented in this paper. The cross-validation method is applied to investigate the generalization capability of the proposed load model. Case studies fully illustrate the accuracy as well as the strong generalization capability of the presented model to describe the load dynamic behavior in the actual power system.

The production-grade load characteristic recorder has been developed and applied around China. The accuracy of the load model developed in this paper has been validated during system operation.

Future work will be focused on the following three aspects. First, the accumulation of the field measurements provides an opportunity to find the statistical law behind the seemingly irrelevant load data. Since all load models can only be built on limited data, the model that captures the inner law of the randomly varied load would be the best one. Thus, the statistic learning theory [28] and techniques on model building based on a small data set [29] will be explored in load modeling practice. Also the statistical law revealed by oceans of data will be applied as guidance on the measurement-based load modeling process.

Second, the general load characteristic at sub-transmission voltage levels will be investigated. The load model so far built has been on the distribution voltage level; however, further work is needed on the aggregated load characteristic on the higher voltage levels. The measurement-based load modeling procedure can be extended to solve this problem; nevertheless, the appropriate model structure to describe the general load characteristic on the sub-transmission voltage level needs to be studied [30].

Third, a global load model will be researched. Since the load may take quite different characteristics under extreme system operating conditions, such as situations near voltage collapse point or when a dramatic voltage dip sustains too long, a global load model may be investigated to adapt to all possible system operating scenarios. The idea of global control [31] will be extended around a global load model and hybrid systems' ideas toward more comprehensive stability control.

The work reported in this paper is just an initial step toward understanding the load better. With more data recorded, more on the load characteristics is expected to be found.

\section{REFERENCES}

[1] C. Concordia and S. Ihara, "Load representation in power systems stability studies," IEEE Trans. Power App. Syst., vol. PAS-101, no. 4, pp. 969-977, Apr. 1982.
[2] J. M. Undrill and T. F. Laskowski, "Model selection and data assembly for power system simulation," IEEE Trans. Power App. Syst., vol. PAS101, no. 9, pp. 3333-3341, Sep. 1982.

[3] R. H. Craven and M. R. Michael, "Load representations in the dynamic simulation of the Queensland Power System," J. Elect. Electron. Eng., vol. 3, no. 1, pp. 1-7, 1983.

[4] IEEE Task Force on Load Representation for Dynamic Performance, "Load representation for dynamic performance analysis," IEEE Trans. Power Syst., vol. 8, no. 2, pp. 472-482, May 1993.

[5] IEEE Task Force on Load Representation for Dynamic Performance, "Bibliography on load models for power flow and dynamic performance simulation," IEEE Trans. Power Syst., vol. 10, no. 1, pp. 523-538, Feb. 1995.

[6] J. V. Milanovic and I. A. Hiskens, "Effects of load dynamics on power system damping," IEEE Trans. Power Syst., vol. 10, no. 2, pp. 1022-1028, May 1995.

[7] IEEE Task Force on Load Representation for Dynamic Performance, "Standard load models for power flow and dynamic performance simulation," IEEE Trans. Power Syst., vol. 10, no. 3, pp. 1302-1313, Aug. 1995.

[8] I. A. Hiskens and J. V. Milanovic, "Load modeling in studies of power system damping," IEEE Trans. Power Syst., vol. 10, no. 4, pp. 1781-1788, Nov. 1995.

[9] A. Gebreselassie and J. H. Chow, "Investigation of the effects of load models and generator voltage regulators on voltage stability," Int. J. Elect. Power Energy Syst., vol. 16, no. 2, pp. 83-89, 1994.

[10] P. Kundur, Power System Stability and Control. New York: McGrawHill, 1993

[11] General Electric Company, "Load Modeling for Power Flow and Transient Stability Computer Studies," EPRI, Rep. EL-5003, vol. 1-4, Jan. 1987.

[12] W. W. Price, K. A. Wirgau, A. Murdoch, J. V. Mitsche, E. Vaahedi, and M. A. El-kady, "Load modeling for load flow and transient stability computer studies," IEEE Trans. Power Syst., vol. 3, no. 1, pp. 180-187, Feb. 1988.

[13] H. Renmu and A. J. Germond, "Dynamic model for load model parameters identification based upon measurement in the field," in Proc. 8th Power System Computation Conf., Aug. 1984, pp. 858-866.

[14] H. Renmu, "Establishment of the power system load model," in Proc. CSEE, vol. 12, Nov. 1992, pp. 33-40.

[15] M. Daqiang and J. Ping, "A novel approach to dynamic load modeling," IEEE Trans. Power Syst., vol. 4, no. 2, pp. 396-402, May 1989.

[16] C. J. Lin, Y. T. Chen, H. D. Jiang, and J. C. Wang, "Dynamic load models in power systems using the measurement approach," IEEE Trans. Power Syst., vol. 8, no. 1, pp. 309-315, Feb. 1993.

[17] J. C. Wang, H. D. Jiang, C. L. Chang, and A. H. Liu, "Development of a frequency-dependent composite load model using the measurement approach," IEEE Trans. Power Syst., vol. 9, no. 3, pp. 1546-1556, Aug. 1994.

[18] J. H. Shi and H. Renmu, "Measurement-based load modeling-model structure," in Proc. IEEE Bologna PowerTech Conf., Bologna, Italy, Jun. 23-26, 2003.

[19] D. N. Kosterev, C. W. Taylor, and W. A. Mittelstadt, "Model validation for the August 10, 1996 WSCC system outage," IEEE Trans. Power Syst., vol. 14, no. 3, pp. 967-979, Aug. 1999.

[20] W.-S. Kao, C.-J. Lin, C.-T. Huang, Y.-T. Chen, and C.-Y. Chiou, "Comparison of simulated power system dynamics applying various load models with actual recorded data," IEEE Trans. Power Syst., vol. 9, no. 1, pp. 248-254, Feb. 1994.

[21] W.-S. Kao, "The effect of load models on unstable low-frequency oscillation damping in Taipower system experience w/wo power system stabilizers," IEEE Trans. Power Syst., vol. 16, no. 3, pp. 463-472, Aug. 2001.

[22] L. Pereira, D. Kosterev, P. Mackin, D. Davies, J. Undrill, and W. Zhu, "An interim dynamic induction motor model for stability studies in the WSCC," IEEE Trans. Power Syst., vol. 17, no. 4, pp. 1108-1115, Nov. 2002.

[23] D. J. Hill, "Nonlinear dynamic load models with recovery for voltage stability studies," IEEE Trans. Power Syst., vol. 8, no. 1, pp. 166-176, Feb. 1993.

[24] D. Karlsson and D. J. Hill, "Modeling and identification of nonlinear dynamic loads in power systems," IEEE Trans. Power Syst., vol. 9, no. 1, pp. 157-166, Feb. 1994.

[25] B. C. Lesieutre, P. W. Sauer, and M. A. Pai, "Development and comparative study of induction machine based dynamic $\mathrm{P}, \mathrm{Q}$ load models," IEEE Trans. Power Syst., vol. 10, no. 1, pp. 182-191, Feb. 1995.

[26] T. Omata and K. Uemura, "Aspects of voltage responses of induction motor loads," IEEE Trans. Power Syst., vol. 13, no. 4, pp. 1337-1344, Nov. 1998. 
[27] O. Nelles, Nonlinear System Identification. New York: SpringerVerlag, 2001.

[28] V. N. Vapnik, The Nature of Statistic Learning Theory. New York: Springer-Verlag, 1995.

[29] P. Radonja, S. Stankovic, and Z. Popovic, "Specific process models derived from extremely small data sets and general process models," in Proc. 7th Seminar Neural Network Applications Electrical Engineering, Sep. 23-25, 2004.

[30] D. J. Hill and I. A. Hiskens, "Modeling, stability and control of voltage behavior in power supply systems," in Proc. IV Symp. Specialists Electric Operational Expansion Planning, Foz do Iguacu, Brazil, May 1994.

[31] Y. Guo, D. J. Hill, and Y. Wang, "Global transient stability and voltage regulations for power systems," IEEE Trans. Power Syst., vol. 16, no. 4, pp. 678-688, Nov. 2001.

He Renmu was born on February 21, 1944. She received the B.S. degree from Tsinghua University, Beijing, China, in 1967 and the Ph.D. degree in electrical engineering from Lausanne Institute of Science and Technology (EPFL), Lausanne, Switzerland, in 1985.

Currently, she is a Professor at North China Electric Power University, Beijing. Her main research interests are power system dynamics, simulation veracity, dynamic load modeling, deregulation, software engineering, and wide area measurements.
Ma Jin (M'06) was born in Taiyuan, Shanxi, China, on March 5, 1975. He received the B.S. and M.S. degrees from Zhejiang University, Hangzhou, China, in 1997 and 2000, respectively, and his Ph.D. degree from Tsinghua University, Beijing, China, in 2004, all in electrical engineering.

Currently, he is with North China Electric Power University, Beijing. His main research interests are load modeling, nonlinear system control, dynamic power system, and power system economics.

David J. Hill (M'76-SM'91-F'93) is now with the Department of Information Engineering, the Australian National University, Canberra, Australia. His research interests are mainly in nonlinear systems and control, stability analysis, power system dynamics and security, and complex system theory and application.

Prof. Hill is a Fellow of the Institution of Engineers, Australia, and a Foreign Member of the Royal Swedish Academy of Engineering Sciences. 